\title{
INVESTIGATION OF THE SPARK CHANNEL OF ELECTRICAL DISCHARGES NEAR THE MINIMUM IGNITION ENERGY
}

\author{
Essmann S. ${ }^{a, *}$, Markus D. ${ }^{a}$, MAas U. $^{b}$ \\ ${ }^{a}$ Physikalisch-Technische Bundesanstalt (PTB), Bundesallee 100, 38116 Braunschweig \\ ${ }^{b}$ Karlsruhe Institute of Technology (KIT), Institute of Technichal Thermodynamics, Engelbert-Arnold-Str. 4, \\ 76131 Karlsruhe \\ * stefan.essmann@ptb.de
}

\begin{abstract}
In this work, we investigate the expansion of the hot gas kernel and pressure wave induced by electrical discharges near the minimum ignition energy experimentally by means of a schlieren setup and numerically through one-dimensional simulations. The effects of discharge energy and energy density on the expansion are discussed. Via comparison of experimental values with numerical simulations, an estimate of the overall losses of the discharge is presented.
\end{abstract}

Keywords: electrical discharge, minimum ignition energy, schlieren.

\section{Introduction}

Electrical discharges are an ignition source that frequently occur in practical applications. In the safety relevant energy range close to the minimum ignition energy (MIE) which - depending on the burnable gas - ranges from $20 \mu \mathrm{J}$ to about $1 \mathrm{~mJ}$, several loss processes play an important role [1. They include external (ohmic) losses, heat transfer to the electrodes, radiation, and losses due the generation of a shock or pressure wave [2]. During the discharge substantial fractions of the electrical energy are injected into a fairly small gas volume in the order of $0.1 \mathrm{~mm}^{3}$ through plasma processes within some $10 \mathrm{~ns}$, leading to a fast increase in temperature [3 6]. The high temperature and pressure lead to a rapid expansion of this cylindrical kernel. Also, a pressure wave forms at the kernel perimeter after several $100 \mathrm{~ns}$. The temporal evolution of the radii of the pressure wave and the kernel reveals information about characteristics of the discharge such as its energy. Through a comparison of experimentally obtained radii with numerical simulations the underlying numerical models can be validated. The goal of this study is to learn about the effects of energy and energy density variation on the expansion of the kernel and pressure wave after electrical discharges near the minimum ignition energy. From the comparison between experiments and simulations, an estimate of the overall losses (or efficiency) for this type of discharge shall be obtained. The experimental determination of radii is done by means of a schlieren setup. They are then compared to radii extracted from one-dimensional gas dynamics simulations. Ultimately, a better understanding of ignition by electrical discharges will help to set standards concerning the design of explosion proof devices in safety relevant scenarios which are based on a scientific approach.

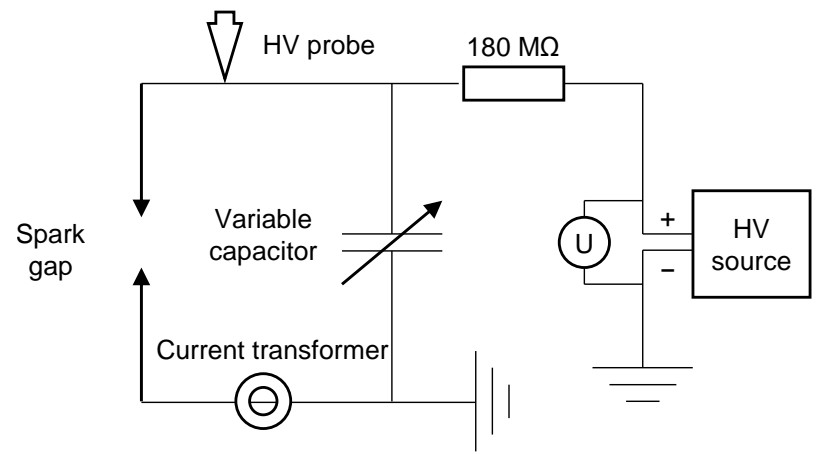

Figure 1. Experimental setup, electrical part.

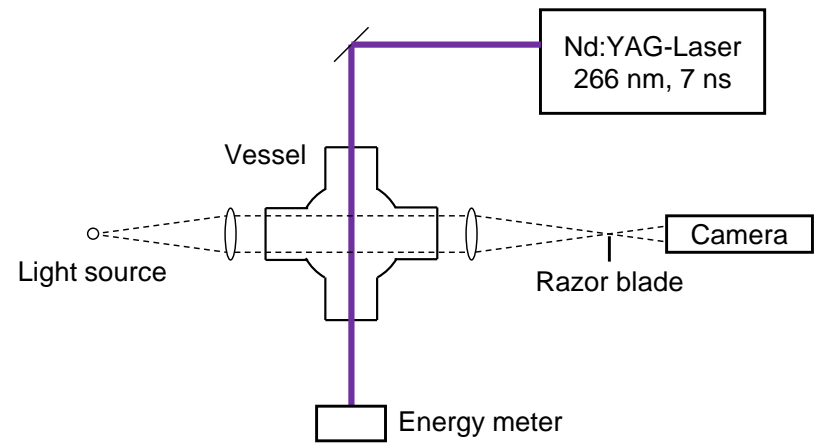

Figure 2. Experimental setup, optical part (top view).

\section{Experiment}

High voltage capacitive discharges were generated between tungsten rods with rounded tips $(2.4 \mathrm{~mm}$ diameter) in a cylindrical vessel. The vessel $(140 \mathrm{~mm}$ diameter, $110 \mathrm{~mm}$ height) had flanges on four sides and was optically accessible through quartz glass windows ( $55 \mathrm{~mm}$ diameter). Prior to each experiment the vessel was flushed with dried air five times its volume to ensure there was no moisture. All experiments were carried out at atmospheric pressure (1 bar \pm 40 mbar) and room temperature $\left(21.5 \pm 1.0^{\circ} \mathrm{C}\right)$. 


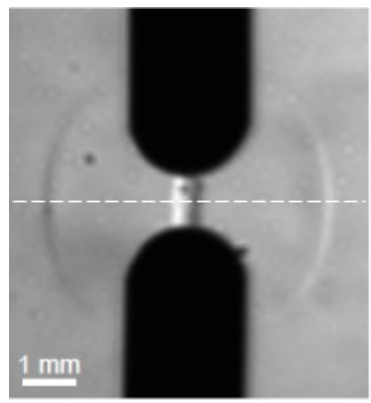

Figure 3. Schlieren image of the kernel and pressure wave taken $6.7 \mu$ s after a discharge in air. The discharge energy was $87.0 \mu \mathrm{J}$ at $1.0 \mathrm{~mm}$ electrode distance.

Figure 1 shows the test setup. A high voltage source (FUG HCP 35-35000) was used to charge a variable vacuum capacitor (3-30 pF, Jennings CADC-30-10S) across a $180 \mathrm{M} \Omega$ resistor. The voltage and current were measured with a high voltage probe (Tektronix P6015A) and a current transformer (Bergoz CT-B5.0), respectively, and recorded on an oscilloscope (Yokogawa DLM6054). Schlieren images of the kernel and pressure wave were taken at different times after the discharge. The schlieren setup shown in fig. 2 consisted of a spark flash lamp (Nanolite KL-L) as the light source, two $500 \mathrm{~mm}$ condenser lenses, a razor blade as the schlieren edge, and a CCD camera $(\mathrm{LaV}$ ision Imager ProPlus 2M) for recording the images. The flash lamp had a pulse width of less than $25 \mathrm{~ns}$, therefore freezing the position of the pressure wave on the image. The spatial resolution of the images was $50 \mathrm{pixel} / \mathrm{mm}$.

The breakdown voltage depends on the pressure, the gas mixture, and the electrode distance. When the applied voltage is larger than the breakdown voltage, discharges occur randomly if free electrons are present in the inter-electrode region, e.g. due to background radiation. When we triggered the measurement instruments based on a rise in the current signal across the electrode gap, intrinsic delays of the instruments made it impossible to take schlieren images at times earlier than $6.5 \mathrm{us}$ after the discharge. In order to investigate this time interval, we triggered the discharges by means of laser radiation. A frequency quadrupled Nd:YAG laser beam (wavelength $266 \mathrm{~nm}, 5-7 \mathrm{~ns}$ pulse duration) was sent through the discharge volume orthogonally to the schlieren setup. The laser beam partially hit the electrode tips where due to the photoelectric effect electrons were emitted. In addition, electrons may become free via multi-photon ionisation [7. The free electrons were accelerated in the electric field and started an electron avalanche. We applied a voltage below the natural breakdown voltage so that no discharged occurred due to the electric field. Then, a laser pulse was fired, generating free electrons in the discharge region and therefore leading to a precisely timed discharge.

Figure 3 shows a sample schlieren image. We can

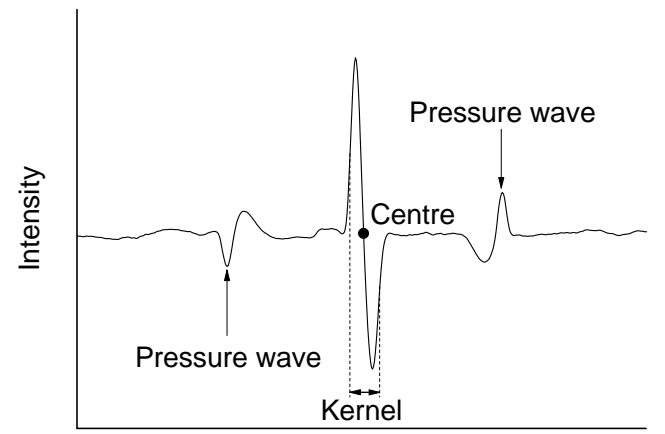

Radial Coordinate

Figure 4. Intensity profile across the dashed line in fig. 3. The kernel radius $r_{\mathrm{K}}$ was defined as the full width at half maximum of the inner extrema while the pressure wave radius $r_{\mathrm{PW}}$ was determined via the outer maximum (right) and minimum (left).

\begin{tabular}{lcc}
\hline \hline Gas Mixture & $L_{\mathbf{s}} / \mathbf{~ m m}$ & $E_{\mathbf{s}} / \mathbf{\mu J}$ (avg.) \\
\hline Dried air & 0.5 & $31.1-159$ \\
Dried air & 1.0 & $89.9-462$ \\
Dried air & $0.5-1.5$ & 249 \\
\hline \hline
\end{tabular}

Table 1. Experimental parameters. $L_{s}$ : Electrode distance (spark channel length), $E_{s}$ : Discharge energy.

observe the cylindrical, hot gas kernel between the electrodes as well as the approximately spherically expanding pressure wave. From the corresponding radial intensity profile their radial positions $r_{\mathrm{K}}$ and $r_{\mathrm{PW}}$ were determined (fig. 4, see caption for details).

In order to work out the effect of discharge energy and energy density on the expansion of the kernel and pressure wave, we carried out experiments in dried air for varying energy $E_{\mathrm{s}}$ and electrode distances $L_{\mathrm{s}}$. Table 1 list details of the parameters. A set of 15 single shot images was taken for every investigated time step, ranging from few ns up to $1 \mathrm{~ms}$ after the discharge. We assumed the discharge energy $E_{\mathrm{s}}$ to be equal to that stored on the capacitor prior to the discharge,

$$
E_{\mathrm{s}}=\frac{1}{2} C U^{2}
$$

where $C$ represents the capacity of the setup (including stray capacitance) and $U$ denotes the voltage before breakdown.

\section{Simulation}

Only the most important parameters of the numerical simulation are given here. For a more detailed description, the reader is kindly referred to $[8$. Onedimensional numerical simulations of the expansion process were performed using the program INSFLA [9. Plasma effects were not modelled, hence, the local gas heating inside the discharge region was considered 
using the energy deposition term [10]

$$
q= \begin{cases}\frac{D_{\mathrm{s}}}{t_{\mathrm{s}}} \exp \left(-\left(\frac{r}{r_{0}}\right)^{8}\right) & \text { for } 0 \leq t \leq t_{\mathrm{s}} \\ 0 & \text { for } t>t_{\mathrm{s}}\end{cases}
$$

where $D_{\mathrm{s}}=E_{\mathrm{s}} /\left(L_{\mathrm{s}} \pi r_{0}^{2} \frac{1}{4} \Gamma\left(\frac{1}{4}\right)\right)$ is the spark energy density deposited during the spark duration $t_{\mathrm{s}}$. $r_{0}$ and $L_{\mathrm{s}}$ represent the spark radius and the spark channel length, respectively.

\section{Results}

\subsection{Effect of energy and energy density}

Figure 5 shows a series of schlieren images which illustrate the expansion of the kernel and the pressure wave after a discharge. The rapid expansion of the kernel can be observed within the first microsecond. After $0.45 \mu \mathrm{s}$, the kernel and pressure wave become distinguishable. The pressure wave travels at $\mathrm{Ma} \approx 1$, its shape changing from cylindrical to spherical as it propagates outward. At $7.5 \mu$ s the kernel begins to deform, its centre part becoming noticeably thinner. Eddies start to form at the electrode tips which pull apart the kernel centre and thus smear it (200-1000 $\mu \mathrm{s})$.

If the pressure wave radius $r_{\mathrm{PW}}$ (cf. fig. 4) was plotted as a function of time, an approximately straight line would be obtained. Its slope corresponds to the sound speed $c$. In order to pronounce the differences in plots of different energies, in the following figures we plot the reduced pressure wave radius $r_{\mathrm{PW}}^{*}=r_{\mathrm{PW}}-c t$ which is the pressure wave radius reduced by the radius of a sound wave starting at $r(t=0)=0$. For the experiments in dried air at $21.5^{\circ} \mathrm{C}, c=344 \mathrm{~m} \mathrm{~s}^{-1}$. Figure 6 shows of the evolution of the kernel and pressure wave, obtained experimentally in dried air at $1.0 \mathrm{~mm}$ electrode distance for four discharge energies (left column). This is compared to numerically obtained values (right column). Here and in the following graphs, each experimental data point represents the average of 15 measurements while the error bars indicate their standard deviation. In the experiments, the kernel radius $r_{\mathrm{K}}$ rapidly increases from 0.2 to $1 \mu \mathrm{s}$ after the discharge. This expansion is more intense as the discharge energy increases. At $0.5 \mu$ s the pressure wave separates from the kernel and travels outwards at $\mathrm{Ma}=1.0-1.1$. At $1 \mu \mathrm{s}$ the first (pressure driven) phase of kernel expansion ends and the radius becomes smaller again (but only in the central region, cf. fig. 5). At 4-5 us the second phase of kernel growth begins which is not pressure driven but due to flow. Both kernel and pressure radii at a fixed time increase with energy due to increased pressure and temperature in the discharge region. The same trends can be observed in the simulations (right column of fig. 6). However, the shape of the curves is different from that in the experiment. The kernel expansion cannot be split into two phases, unlike in the experiment. This is due to two-dimensional effects which are not covered in the simulation. Therefore, a comparison between experiment and simulation is only feasible up to $5 \mu \mathrm{s}$ after the discharge. At that time, in the simulation, the kernel has reached its final size and no further growth is observed. The plot of reduced pressure wave radii shows a slight inclination $(\mathrm{Ma}>1)$. The radius increases with discharge energy, a trend that is reproduced by the simulation. For times later than $2 \mu \mathrm{s}$, there is considerable scatter in the radii taken from the numerical simulation. This is due to the relatively coarse numerical grid at larger distances from the centre. Therefore, pressure wave radii obtained from the experiment and simulation should only be compared up to $2 \mu$ s after the discharge.

The effect of energy density in the experiment is shown in fig. 7. Here, the discharge energy was kept constant while the electrode distance was varied. The discharge radius, i.e. the kernel radius at very early times, does not change with energy density within the margin of error. Therefore, the energy density is inversely proportional to the electrode distance in this case. Qualitatively, the same characteristics as in fig. 6 may be observed, though the effect of energy density variation is less distinct than that of energy variation.

\subsection{Initial radius}

The initial radius $r_{0}$ is an important parameter in the numerical simulations, since the energy source term contains $r_{0}^{2}$. Therefore, small changes in $r_{0}$ will have a significant effect on the evolution of the kernel and pressure wave. Figure 8 shows the effect of discharge energy on the initial radius. We calculated $r_{0}$ as the average of kernel radii from $0-200 \mathrm{~ns}$ as in this time interval the radius is approximately constant. We observe a clear dependence of $r_{0}$ on the discharge energy such that an increase in energy will yield a larger initial radius. As stated before, the energy density has no apparent effect on the initial radius.

Figure 9 shows the effect of the initial radius on the numerical simulation results. We varied $r_{0}$ from 100 to $150 \mu \mathrm{m}$ while keeping all other simulation parameters constant $\left(E_{\mathrm{s}}=111 \mu \mathrm{J}, L_{\mathrm{s}}=0.5 \mathrm{~mm}\right)$, resulting in a smaller energy density $D_{\mathrm{s}}$. The simulations indicate that larger energy density leads to higher maximum temperature and pressure in the discharge region. Nevertheless, the initially smaller kernels will not catch up to the larger ones as the discharge energy is equal in all three cases. This result shows that a precise knowledge of the initial radius is crucial when simulations and experiments are compared quantitatively.

\subsection{Estimation of discharge efficiency}

Since the kernel and pressure wave are affected by the discharge energy, comparing the experimental data with numerical simulations could yield an estimate of the overall losses. Here, we consider the efficiency $\eta$ of the discharge, which is simply a factor in eq. 2 , so the effective source term including losses becomes $q_{\mathrm{eff}}=\eta q, 0 \leq \eta \leq 1$ [4]. It is an integral value that 


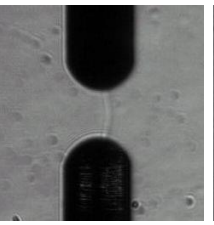

$0.031 \mu \mathrm{s}$

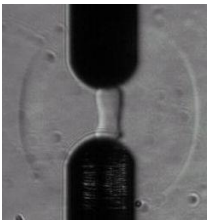

$7.5 \mu \mathrm{s}$

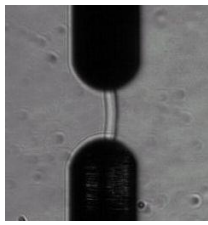

$0.15 \mu \mathrm{s}$

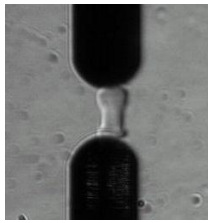

$15 \mu \mathrm{s}$

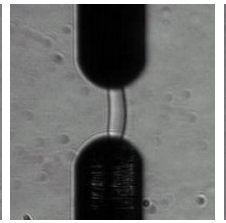

$0.30 \mu \mathrm{s}$

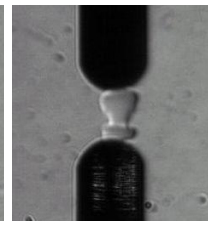

$30 \mu \mathrm{s}$

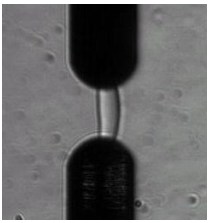

$0.45 \mu \mathrm{s}$

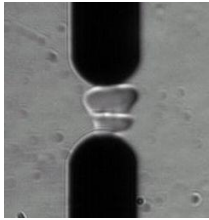

$100 \mu \mathrm{s}$

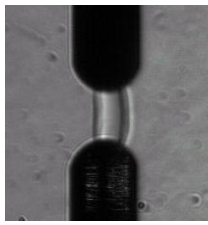

$0.96 \mu \mathrm{s}$

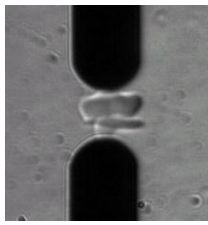

$200 \mu \mathrm{s}$

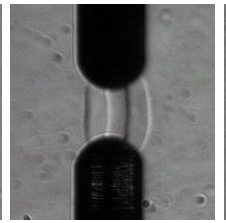

$2.0 \mu \mathrm{s}$

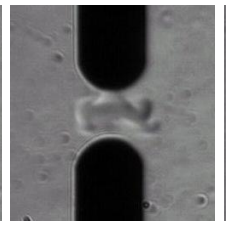

$500 \mu \mathrm{s}$

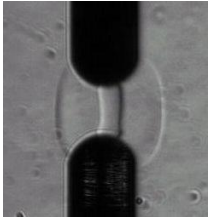

$4.0 \mu \mathrm{s}$

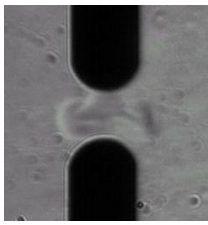

$1000 \mu \mathrm{s}$

Figure 5. Schlieren series of the kernel and pressure wave after an electrical discharge in 5.2 vol.-\% propane/air $\left(L_{\mathrm{s}}=1.7 \mathrm{~mm}, E_{\mathrm{s}}=241 \mu \mathrm{J}\right)$.
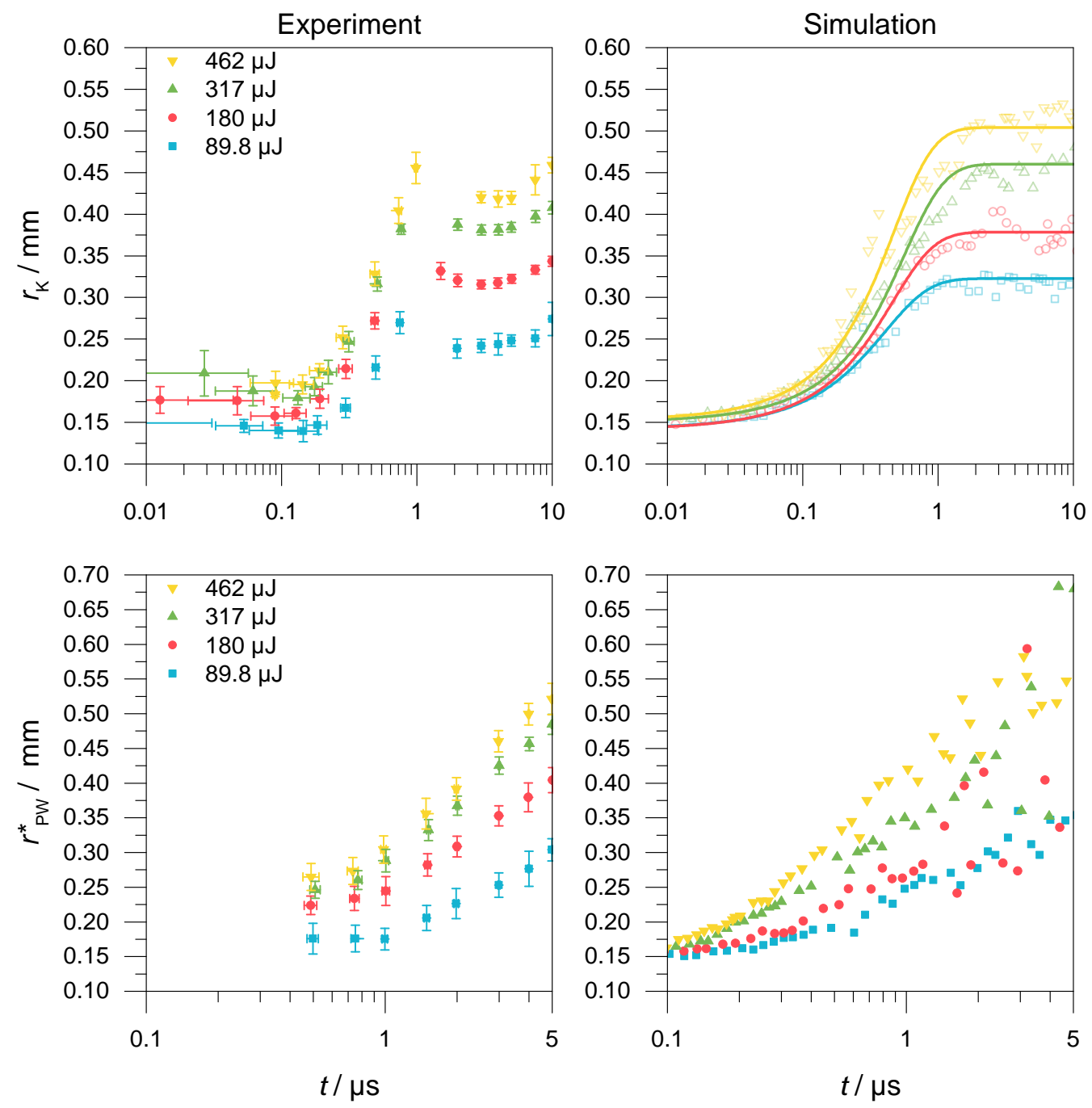

Figure 6. Kernel radius (top row) and reduced pressure wave radius (bottom row) as a function of time after a discharge at $1.0 \mathrm{~mm}$ electrode distance for varying discharge energy. Comparison of experiments (left column) with simulations (right column). The initial radius was $r_{0}=150 \mu m$ in all simulations. Only some error bars are shown in the bottom right plot. 

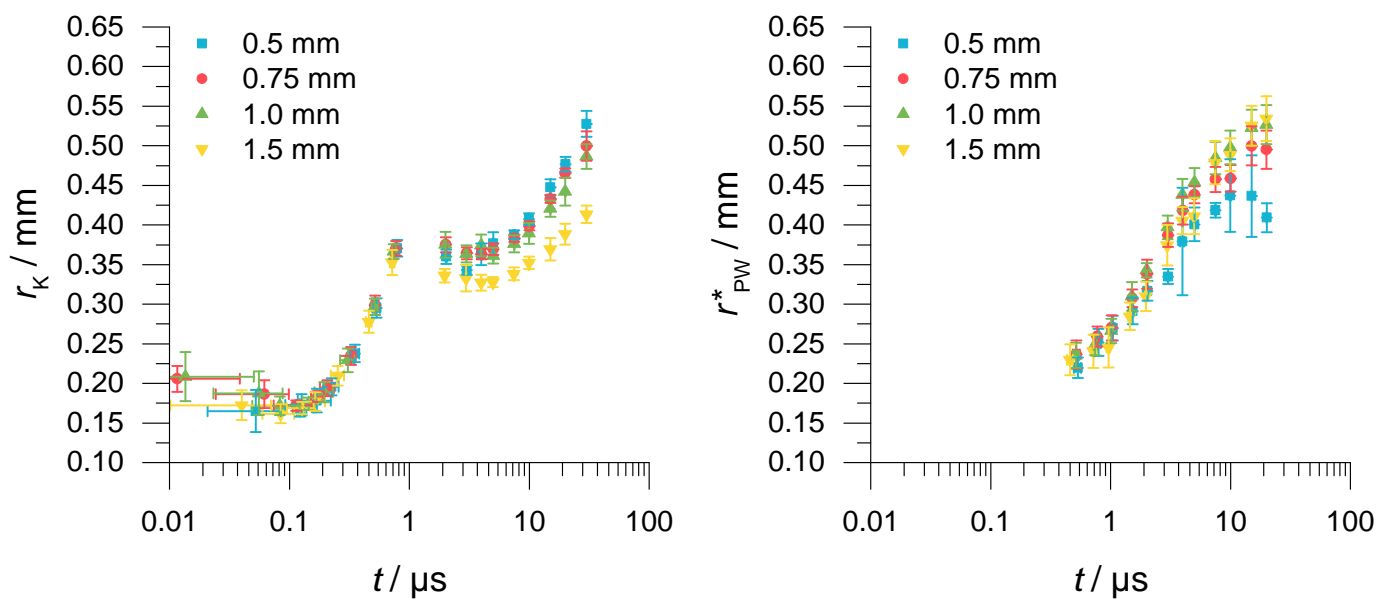

Figure 7. Experimentally obtained kernel radius (left) and reduced pressure wave radius (right) as a function of time after a discharge at constant energy $E_{\mathrm{s}}=249 \pm 5 \mu \mathrm{J}$ for varying electrode distance.

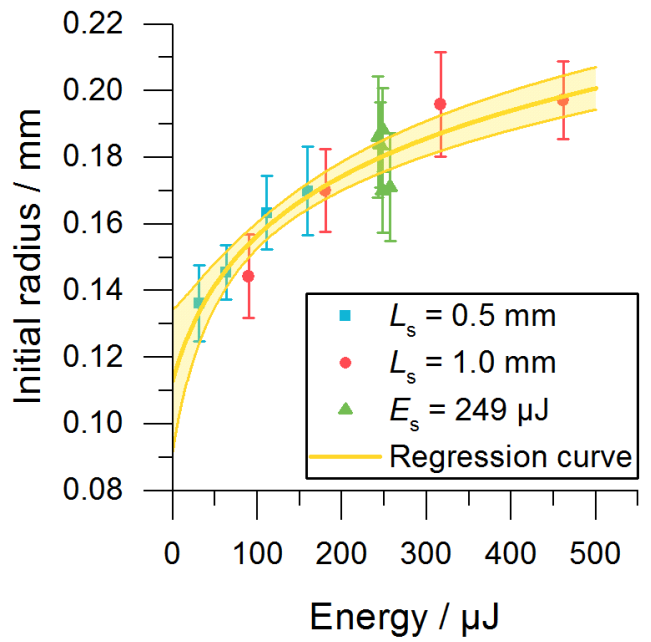

Figure 8. Initial radius $r_{0}$ obtained from the experiments as a function of discharge energy. The regression curve is logarithmic: $r_{0}=b \ln \left(E_{\mathrm{s}}-a\right)$, $R^{2}=0.91$. The $95 \%$ confidence band is also shown.

takes into account all kinds of losses that are not numerically modelled, such as radiation, heat transfer to the electrodes, and external (e.g. ohmic) losses. In addition, $\eta$ takes into account the fraction of input energy that does not rapidly heat the gas volume, for example the relaxation of vibrationally excited molecules which takes significantly longer than $1 \mu \mathrm{s}$ in air at atmospheric pressure [5]. Therefore, this portion of energy does not contribute to the early stages of kernel and pressure wave expansion.

Figure 10 shows the comparison of experimentally obtained results with numerical simulations for different values of $\eta$. The time interval was constrained to the limits described in section 4.1 .

\section{Conclusions}

The expansion of the kernel and pressure wave induced by electrical discharges near the minimum ignition energy was investigated. We used the schlieren tech-

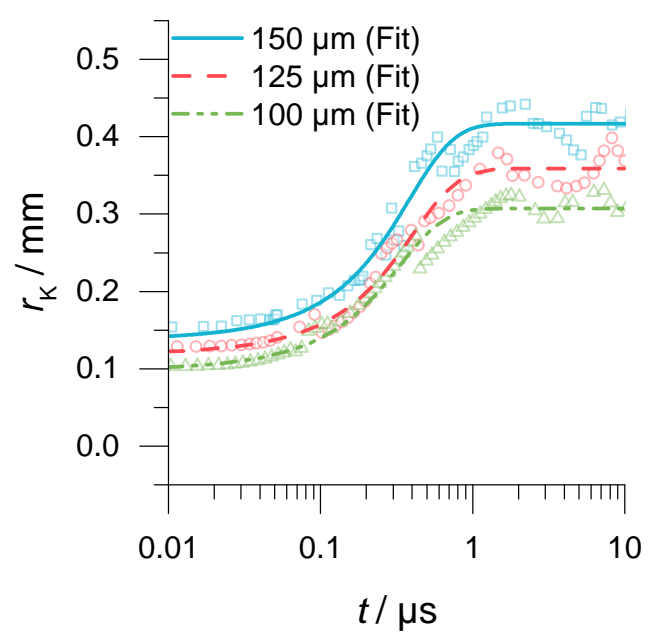

Figure 9. Effect of changes in the initial radius $r_{0}$ on the kernel evolution in numerical simulations. All other simulation parameters were kept constant $\left(E_{\mathrm{s}}=\right.$ $111 \mu J, L_{\mathrm{s}}=0.5 \mathrm{~mm}$ ).

nique to obtain the radii at different points in time. The discharges were laser-triggered so as to make the time interval $t<6.5 \mu \mathrm{s}$ accessible. The effects of discharge energy and energy density on the expansion were investigated. We found that both $r_{\mathrm{K}}$ and $r_{\mathrm{PW}}$ at a certain time increase as the energy rises while the effect of energy density is much less pronounced. Onedimensional numerical simulations of the expansion process were performed and yielded the same trends as in the experiment. A comparison of the radial position between experiment and simulation can yield the efficiency of the discharge. However, as of now it is a rough estimate because there are uncertainties in the initial radius $r_{0}$ which has a great influence on the simulation.

Therefore, future work will be concerned with the relation of the initial radius determined from experiments and that used in the simulation. We will investigate different definitions of the source term 

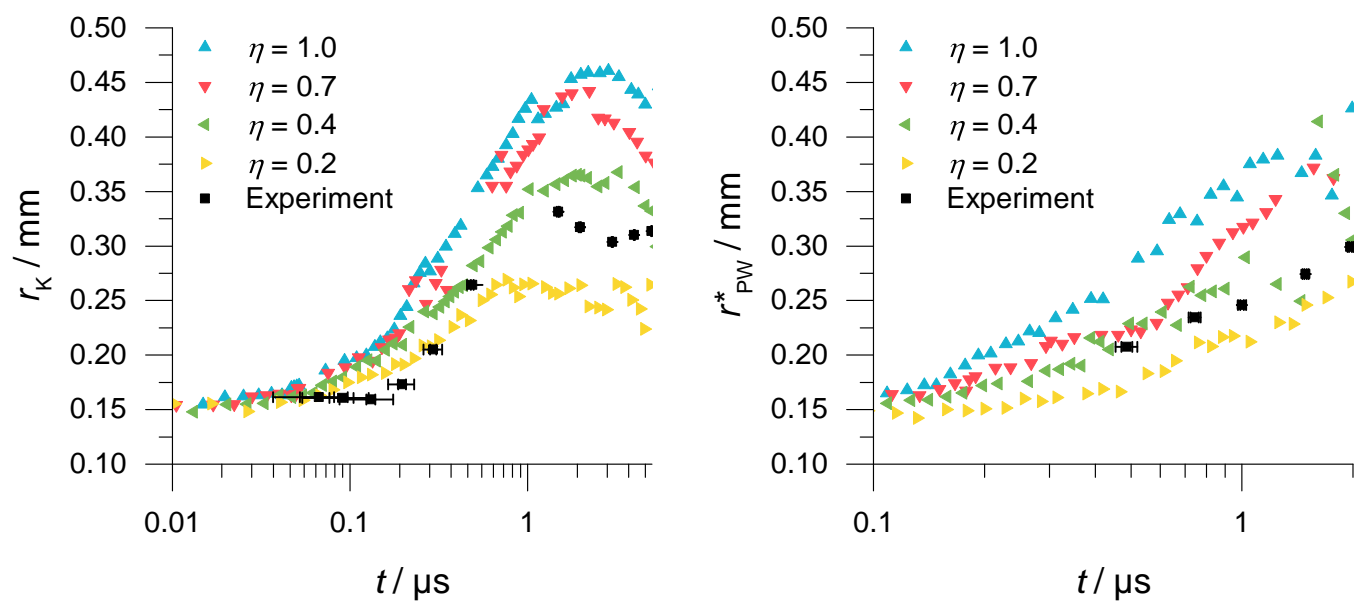

Figure 10. Kernel radius (left) and reduced pressure wave radius (right) as a function of time after a discharge at $0.5 \mathrm{~mm}$ electrode distance and $159 \mu \mathrm{J}$ discharge energy. Comparison with radii obtained from numerical simulations with varying discharge efficiency $\left(r_{0}=150 \mu \mathrm{m}\right)$.

in the simulations. In addition, we plan to perform two-dimensional simulations in order to capture fluid dynamics effects and heat transfer to the electrodes. Experiments concerning the investigated processes in mixtures of air with the burnable gases hydrogen, ethylene, and propane at their respective minimum ignition energy configurations have already been performed and will be compared to simulations as well.

\section{Acknowledgements}

This work was supported by the Deutsche Forschungsgemeinschaft (DFG) under grant FOR 1447.

\section{References}

[1] D. R. Ballal and A. H. Lefebvre. The influence of spark discharge characteristics on minimum ignition energy in flowing gases. Combustion and Flame, 24(0):99-108, 1975.

[2] Rolf K. Eckhoff and Werner Olsen. A new method for generation of synchronized capacitive sparks of low energy. reconsideration of previously published findings. Journal of Electrostatics, 68(1):73-78, 2010.

[3] D. L. Rusterholtz, D. A. Lacoste, G. D. Stancu, D. Z. Pai, and C. O. Laux. Ultrafast heating and oxygen dissociation in atmospheric pressure air by nanosecond repetitively pulsed discharges. Journal of Physics D: Applied Physics, 46(46):464010, 2013.

[4] D. A. Xu, M. N. Shneider, D. A. Lacoste, and C. O. Laux. Thermal and hydrodynamic effects of nanosecond discharges in atmospheric pressure air. Journal of Physics D: Applied Physics, 47(23):235202, 2014.

[5] N. L. Aleksandrov, S. V. Kindysheva, M. M. Nudnova, and A. Yu Starikovskiy. Mechanism of ultra-fast heating in a non-equilibrium weakly ionized air discharge plasma in high electric fields. Journal of Physics D: Applied Physics, 43(25):255201, 2010.

[6] N. A. Popov. Investigation of the mechanism for rapid heating of nitrogen and air in gas discharges. Plasma Physics Reports, 27(10):886-896, 2001.
[7] W. B. Lewis and W. R. Wadt. Laser-induced fluorescence in $\mathrm{n} 2$ and $\mathrm{n} 2+$ by multiple-photon excitation at $266 \mathrm{~nm}$. Chemical Physics Letters, 78(2):266-269, 1981.

[8] Stefan Essmann, Detlev Markus, and Ulrich Maas. Investigation of the pressure wave and hot gas kernel induced by low energy electrical discharges. In 25th International Colloquium on the Dynamics of Explosions and Reactive Systems, 2015.

[9] U. Maas and J. Warnatz. Ignition processes in hydrogen-oxygen mixtures. Combustion and Flame, 74(1):53-69, 1988.

[10] Andreas Dreizler, Steffen Lindenmaier, Ulrich Maas, J. Hult, M. Aldén, and C. F. Kaminski. Characterisation of a spark ignition system by planar laser-induced fluorescence of oh at high repetition rates and comparison with chemical kinetic calculations. Applied Physics B: Lasers and Optics, 70(2):287-294, 2000. 